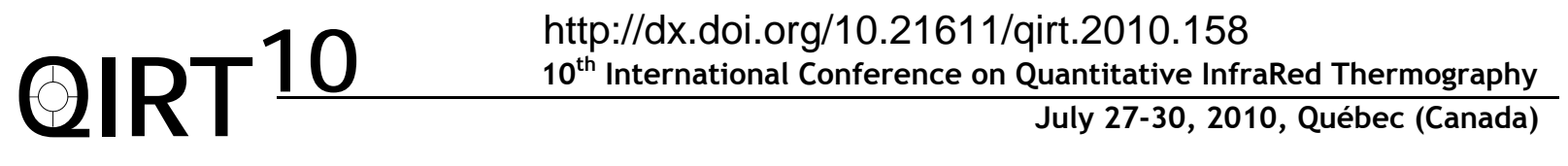

\title{
Thermographic Assessment of Cold Stimulation on Autonomic Vascular Reactions
}

by S. Edwards

\author{
Bioengineering Student, University California Riverside, Riverside California, USA, skedwards@charter.net
}

\begin{abstract}
The objective of this study is to determine the potential usefulness of thermal imaging technology to evaluate and monitor autonomic vascular reactions using a double blind cold water stimulation test. Results were defined among three groups of participants, normal control, smokers, and diabetics. This research established that thermal imagery can be used to effectively detect pre-clinical stages of circulatory problems using a 120x160 resolution imager.
\end{abstract}

\section{Introduction}

To test my hypothesis as to the effectiveness of thermography as a useful tool for assessing autonomic vascular reactions, a cold stimulation test was performed on over 60 participants (Figure 1.) using a radiometric thermal imager. The participants included diabetics, smokers, and a normal/control group (no known health issues).

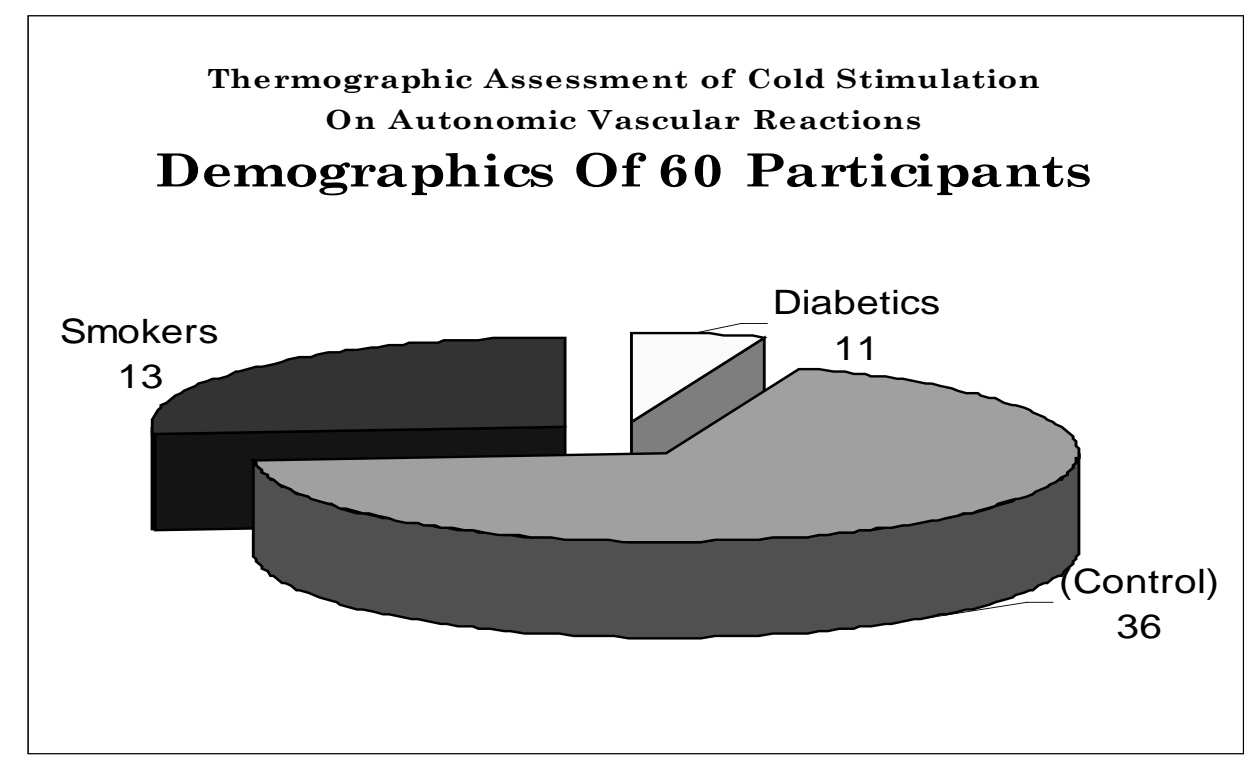

Fig. 1. Demographics of Participants

The test consists of participants placing their left hand in $62^{\circ} \mathrm{F} \pm 2^{\circ}$ water for 20 minutes while the right hand was placed on a wood surface. The participants were also asked to keep the left hand moving while it was submerged in the cool water. Participants were also asked to keep their right hand in a flat comfortable position and to keep movement to a minimum. A thermal image was recorded of the right hand every 5 minutes starting with a preliminary test image. The imager that I used was a Raytek Ti30, resolution $120 \times 160$. 


\section{Discussion}

Prior to analyzing the data, medical history was reviewed and taken into account. Participants in the study were given a corresponding number so that the names remained confidential. Participant's numbers were placed into the appropriate group after a review of their medical history. The groups were: Normal/Control (figure 2.), Diabetics (figure 3.), and Smokers (figure4.). It was noted how long smokers had smoked and how many packs per day (Figure5.). It was also noted how long Diabetics had been diagnosed with Diabetes (Figure 6.), what type, and if they were taking medication for this. The age of the participant was also logged as was if they were male or female.

Three groups of people were clearly defined by the thermal imager. The diabetic group had no visible thermographic finger tip change while the overall average temperature did decline slightly by an average of $2^{\circ} \mathrm{F}$. The smoker group had an extremely mottled and uneven thermographic appearance of all fingers and hand with a slight decline initially in temperature while rising $2^{\circ} \mathrm{F}$ and holding constant until the end of the 20 minute test period. The normal/control group's thermographic assessment of the circulatory system showed that the right hand was significantly affected, with a steady decline in surface temperature as much as $10^{\circ} \mathrm{F}$ (average of $8^{\circ} \mathrm{F} \pm 2^{\circ}$ ) and beginning with very visible thermographic finger tips to almost non visible appearance at the end of the 20 minute test period.

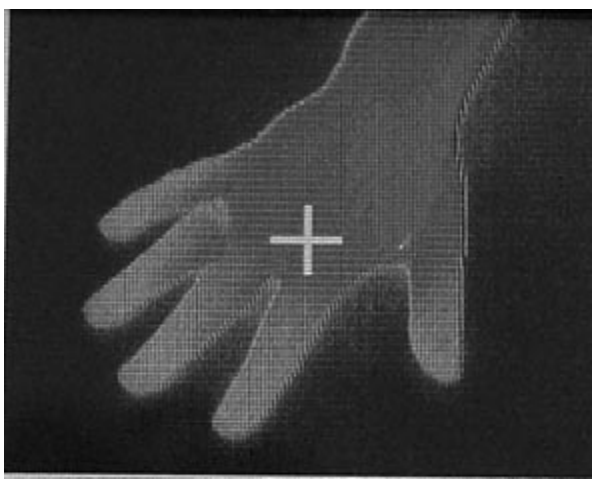

Fig. 2. Normal

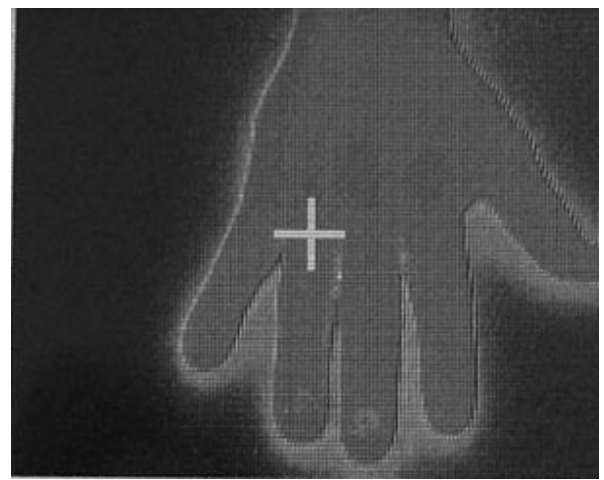

Fig.3. Diabetic

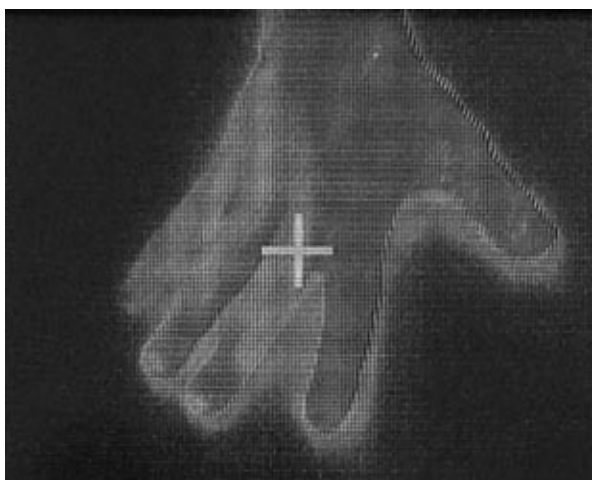

Fig. 4. Smoker

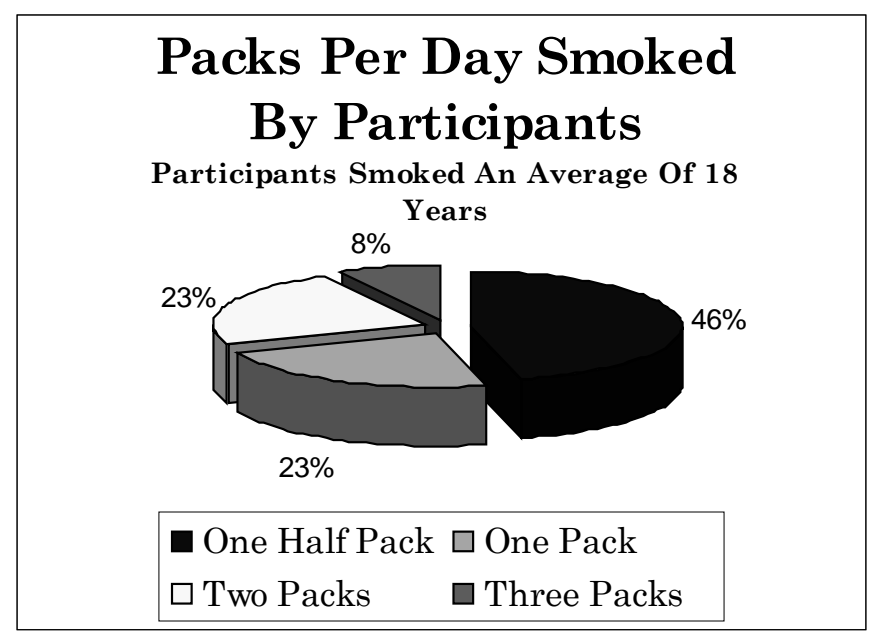

Fig. 5. Packs smoked per day
Thermographic Assessment of Cold Stimulation On Autonomic Vascular Reactions

\section{Years of Diagnosed Diabetes}

Participants Diabetic An Average Of 8 Years

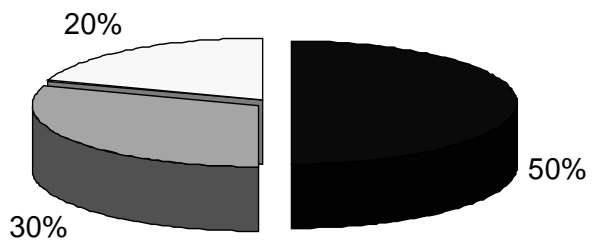

2-5years $\square$ 6-10years $\square$ 13-20years

Fig. 6. Years of diagnosed Diabetes 


\subsection{Image Analyzing Process}

Image Analyzing Process of Images taken with a Raytek Ti30:

To analyze the thermal images taken (Figure 7.), images needed to be downloaded via computer. All five of the participant's images were placed on the same page.

Every dorsal tip finger and dorsal hand average were logged.

A .25" square was drawn around each finger tip (Figure 8.) and hand and temperature pixels were averaged. I logged them in Celsius and Fahrenheit. The temperatures are recorded on the participants hand file. The finger tip averages from each hand plus the upper half of the hand average were again averaged together and entered into Excel resulting in my charts and graphs.

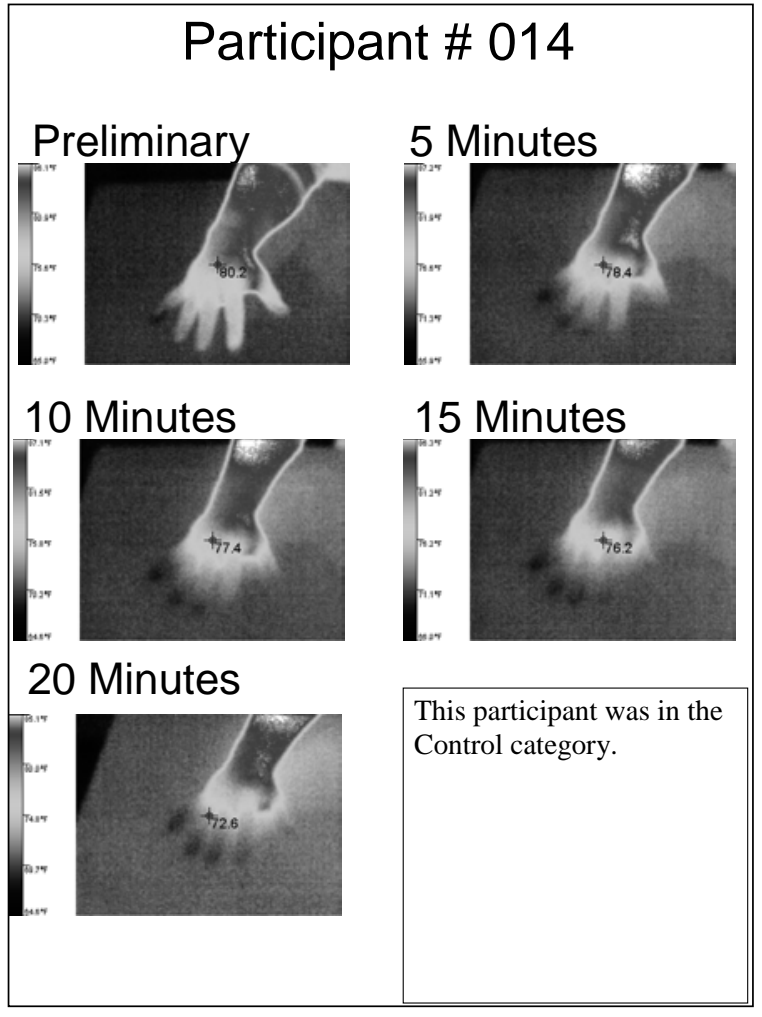

Fig. 7. Participant \#014 Image Log

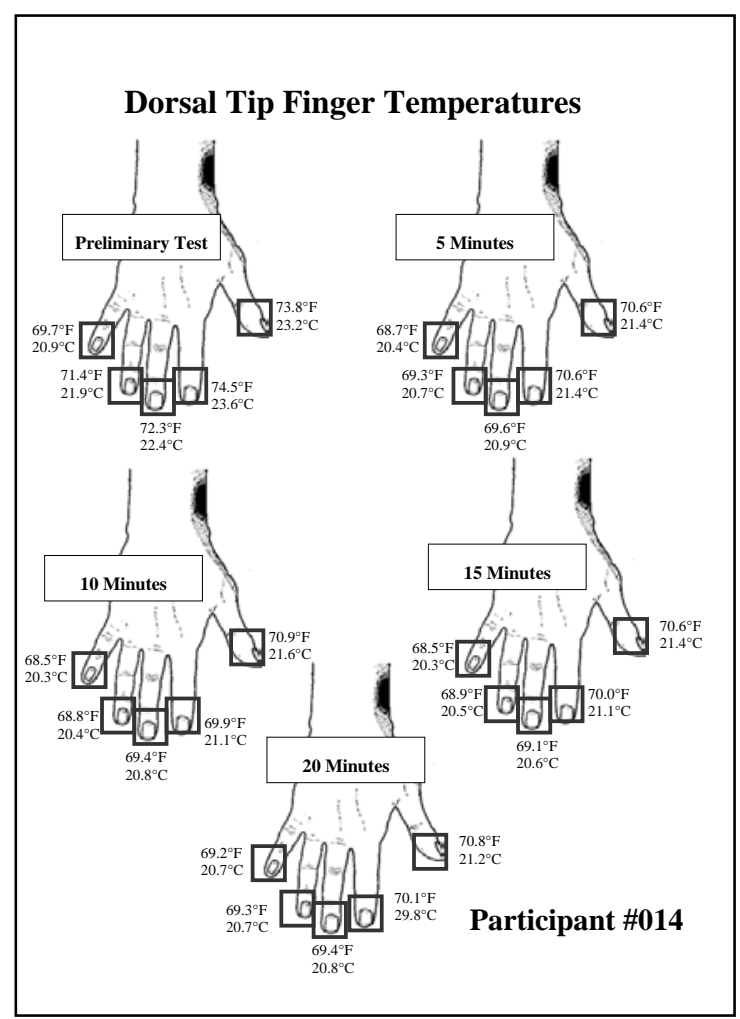

Fig. 8. Participant \#014 Temperature Log 


\section{Conclusion}

In using cold water stimulation to test the autonomic vascular reactions, the results were clearly defined (Figure9.). Using three defined groups amazingly showed clear differences in circulation between each of the groups. The data from the test supports my hypothesis that thermal imaging can be a useful tool for examining circulation. Thermography simply identifies thermal anomalies using an infrared imager.

Smoker's autonomic vascular reactions were very visible using the thermal imager. As with diabetics, the results were also clearly defined and had the most consistent results of all three groups. All diabetics hand patterns were the same. The control group was larger and may have more variables in it yet still showed consistent results.

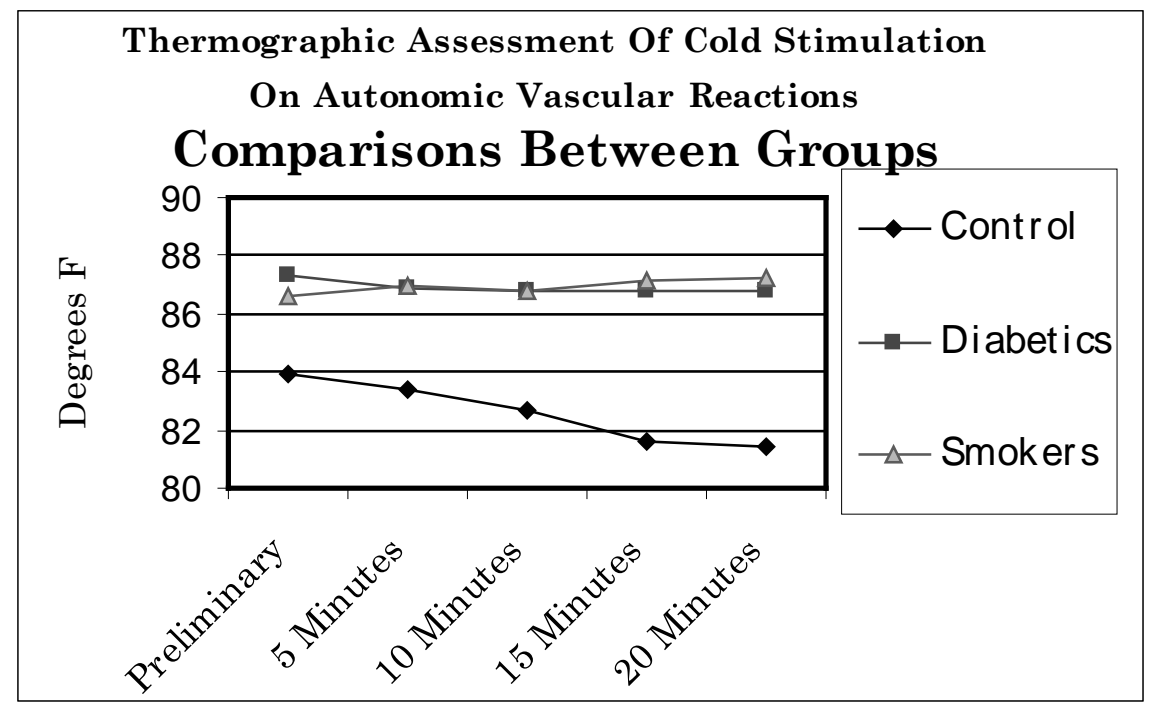

Fig. 9. Comparison between groups

My objective for this test was really to see if I could use a low cost thermal imager normally used in an electrical substation and see if the imager would pick up vascular differences to a cold stimulation test. I had no idea that it this would work or not. I realized that the diabetic participant's thermal patterns did not fluctuate unlike the other two groups. As you well know diabetes is increasing at an alarming rate and I wanted to share my findings. My test not only identified clinically diagnosed participants but identified two participants whose medical history and images matched the diagnosed participants giving surety that they are destined to be diabetic.

Not only was my objective met and significantly impressed the participants with a visual image to be more aware of their health, but most of all using this thermal imaging technique may prove to be the most cost effective solution for third world countries, since only a low cost, mid-performance imager is needed in identifying previously undiagnosed diabetics and determine the damage to their circulatory system. The applications for this type of assessment are wide spread. From early diagnosis of vascular problems to monitoring rehabilitation there are a lot of possibilities for thermographic assessment of vascular reactions. This research proves that thermal imaging can be used effectively to detect pre-clinical stages of circulatory problems. 